Editorial

\title{
Issues in manuscript writing
}

Writing a manuscript is an art of a science......

Publication is kind of documentation to be used for further advancing research. If it is not documented, it is equal to not done, this statement itself highlighting the importance of documentation/publication in the field of scientific research. The RESEARCH word itself self-explanatory and it is defined as researching the elements from excising galaxy. So every researcher searching for something in existing galaxy and whoever documenting/publishing first will get credit. Process of documentation started in Neolithic human period itself. They use to make symbols and identification marks when they are moving one place to another place and that marking are till now identifiable. Now also we can see written scripts in rock temples, pyramids and ancient cultural centers, through this, we can now able to understand the life style of ancient culture and civilization. Neolithic to modern human most common unchanged character observed is documentation, but way of documentation only changing time to time. In modern era, publication is one of the powerful tool for documentation and also electronic publication is having major contribution in the field of science and medicine.

In the past few years, while managing articles submitted to Journal of Young Pharmacists [www.jyoungpharm.org] and Journal of Pharmacology and Pharmacotherapeutics [www.jpharmacol.com], we observed wide variety of issues with submitted manuscripts. In this editorial, we would like to share some of our experiences which could help authors to overcome the shortfalls and prepare the manuscript in order to rejections. It is a part of our duty to highlight such issues to audiences/authors and readers to make them understand the problems associated with writing manuscripts to any scientific journals.

\section{Who is an author?}

As per Wikipedia, "An author is the originator of any written work" [1]. Authors play a major role in contributing the growth of science with the original writings that will enhance or encourage the scientific community. Scientific Articles are the most referred source for any research work, hence it play pivotal role in advancing science. While submitting manuscripts, author should make up their mind and prepare the manuscript as per the journal's requirement. Authors should also be quite serious as they are dealing with the learned editors of a journal.

During submission process, authors should be truthful and honest with the dealing editors. Authors should provide all the mandatory details such as conflict of interest (if any), funding sources and other related issues with the manuscript. The order of authors should be well planned, before submitting the manuscript.
Often, we tend to receive many requests from the authors for adding new authors, changing author order etc during or post acceptance or in the stage of peer review process. JYP and JPP has been very strict in dealing with such requests, we have mostly denied such requests from the authors. In case, if two or more authors equally contributed to the research work, it should be clearly mentioned with asterisk stating as "Author XXX and author YYY have equally contributed to the articles". Such notes will give equal authorship to multiple authors and it is widely acceptable in most of the journals. Author should also include copyright form, which is the most important document for processing the manuscripts, without which none of the articles will be processed for peer review or final publication. Copyright forms are usually submitted during initial submission or during peer review process. Editors will not process blind articles unless there is a correct contact details specified in the paper. Some of the additional information could be title, highest degree etc will be mandatory sometimes.

\section{Author related issues}

During manuscript preparations for any particular scientific journal, author is required to read the instructions carefully and prepare the manuscript accordingly. Most of the time, the template for manuscript preparation available with author instructions section, In case, if the template is not available, author can refer to any of the published paper from recent issue of the journal. Majority of errors occur in first initial page of manuscript, abstract etc. The manuscript should include specific title followed by Authors (In the order of their contribution), Affiliation identifying all the mentioned authors, a short running title and corresponding details of communicating author. While mentioning corresponding author, one should take care of providing correct contact details such as postal address, emails (at least two), phone numbers including landline and mobile numbers and fax number, if available. As the contact details are most important to the editor without which editor will not be able to communicate with you related to the submitted articles. Email communication is always preferred in most of the journals, as the current e-generation. As the editor work for journals on honorary basis, it becomes financial burden to communicate either via post or phone. Hence, email is an important and cheaper medium which connects well with editor and authors.

\section{Biomedical issues}

Prior ethical committee permission for pre-clinical animal studies, studies on animal cell lines and clinical studies. In case of clinical studies, the clinical trial must be registered with clinical trial registry (CTR). If trial is not registered with CTR manuscript 
will not be considered for publication. Similarly, if the author is including any medical images of patients (demographical, morphological clinical futures), it is mandatory to submit the patient/study subject informed consent [2].

\section{Issues on biostatistics}

Results of the study must be analyzed using appropriate statistical method. For example comparison of the weekly blood glucose levels for three different groups of patients with one month drug therapy must be analyzed using Two-way ANOVA, but most of time, we noticed authors used One-way ANOVA for comparing more than two variables. Inappropriate statistical method selection will give either false positive or false negative results. The statistical importance or implication on the study has been clearly described in results section. The level of significance, comparison groups, statistical method, type of post-hoc test has to be described properly in footnote of the table.

\section{Issues in tables and images of the manuscript}

The provided images must be clear and in prescribed dimensions. If author fails to provide high resolution images for their manuscript, it will not be considered for including it in the article. Clinical or medical images provided by authors in clinical case reports, the image must be properly masked to respect patient's identity. Hence, whenever author submitting any medical images its mandatory to submit patient informed consent document, if not manuscript will be rejected or retracted form journal at any point of time.

Tables should be labeled properly and the units for each parameter in the table must be given. In some manuscript we observed ' $n$ ' symbol for non-significant values and enter table 'ns' only predominately visible. It's not required to mention 'ns' for non-significant values. In few of the manuscripts, authors have provided their result in both table and graphical forms (histogram/pi diagram), which is usually not required to have results in multiple presentations. The results can be presented either tables or graphs or any of the prescribed standard format/image. We have observed that histograms are included uses multiple color rather, authors can use simple black and white histograms or filled with line, pattern etc.

\section{Citation related issues}

Citation should be appropriate in submitted manuscripts. Inappropriate citation will give/reflect the wrong opinion on specified area of the research. Hence, it is advised that the cited paper should be proper one and author should have gone through the paper which he/she cited in their manuscript. Editors of the journal have all rights to ask for original copies of cited paper. If author fails to submit the copies of cited paper at any point of time (even after publication), the manuscript/paper will be rejected or retracted form the journal. If author citing any published paper by reading the abstract, that can be cited and end of the reference author have mention in close bracket "abstract". If author citing any published papers/resources other than English author should include the language in parenthesis published in reference.

\section{Issues in scientific content}

Scientific content is a heart of any manuscript. The novelty of content will be (even negative findings) will be considered for publication. Whenever we receive the manuscript the initial screening will be done at editor's levels to assess the reliability of results and acceptability of the paper. Further, the manuscripts will be subject to plagiarism checking using ithenticate or any other standard tool.
If article found anywhere (except conference abstract submission) or found copied verbatim, we are forced to reject the manuscript on this basis.

\section{Submission of manuscript related issue}

Duplicate/multiple submission of the same manuscript to different journals are also an unethical practice. Some of the authors using fresh submission for submitting the revised manuscript, it leads to greater confusion by the editors. Hence, it is always required to submit the revised article with the option of submitting revised manuscript in the system.

Some authors submitting their manuscript in different journals at the same time, it is not a healthy scientific publishing practice as observed by most of the editors globally. It would be a waste of scientific time being spent by editors and reviewers. Once the manuscript is been accepted by one of the journal, author tends to write a withdrawal request to editor without mentioning any reason. Authors are requested to respect the scientific time involved (both by editors and reviewers) in refining one's paper. As per JYP and JPP policy, If author wishes to withdraw their manuscript at any stages after submission (except changing of manuscript type) have to pay system management fee i.e., USD 50 or equivalent to Indian Rupees with proper reasoning for withdrawal of manuscript. Recently, we have faced an shocking incidence with one of the accepted paper of J Young Pharm, which is been published in other journals well before publishing in J Young Pharm. Based on this act, we have blacklisted the authors and we have made a decision of retraction of the published paper in J Young Pharm.

\section{Conflict of interest related issues}

We received frequent complaints from some of the authors, regarding missing, non-inclusion of some author name in published papers. Both the journals are now very clear about the author contributions and order of appearance cannot be changed after submission of article to the journal. Even then we are receiving the request for changing the authors name after publication or some of the missing authors raising conflict. From this point onwards if we received any such issues from missing authors, modifying the authors name after publication that particular manuscript will be withdrawn/retracted without any prior notice.

\section{Major reasons for rejection of manuscript in Journal of Young Pharmacist}

- Incomplete manuscript submission (Title page and manuscript page)

- Non submission of signed contributors form

- Ethics committee permission not addressed

- Clinical trials not registered in CTR

- Poor introduction to the topic

- Poor methodology, improper methods and reference selection

- Copied verbatim

- Volume of fluid collection (excided recommended volume of fluid collection)

- Statistical issues

- Split publication

- Dual publication

- Conflict of interest

- Discussion not as per IMRAD format

- Improper discussion writing/language errors

- Not responding to reviewers/editors comments

- Conflict of interest 
- Non-submission of revised articles within the deadline

- Inappropriate citations. CONSORT flow chart for clinical studies.

\section{Editor related problems}

In a multidisciplinary journal, Editor will look after all the publication process, however, some of the manuscript topics will be out of editor's subject for wide range of research. Hence editors seek help from associate editors, section editors, editorial board members and reviewers for their expert opinion. Each article will be technically checked and further subjected to blind peer review, wherein the author's identity is hidden to prevent biased peer review.

Due to unavoidable circumstances, not all the time, the reviewers and expert opinions will not be in home on time, hence the peer review process takes its own time to scrutinize the novelty of manuscript/work submitted to journal. Author should cooperate and respect the time taken for the peer review process, repeated emails about manuscript status will make the editors annoyed and frustrated. It is author's responsibility for his manuscript in order to get their articles refined and ready for publication. As per editor point of view, he will be working for the journals on honorary basis and sparing time out of his routine work and he will handle three to ten manuscripts per day. Hence, we are requesting all the authors when submitting the manuscript, be serious on language typographical, tables \& figure citation errors, chronologically cited references and manuscript should strictly follow instructions.

\section{Effective manuscript submission to Journal of Young Pharmacist}

Before submitting manuscript to our journal, plagiarism should be checked to avoid accidental plagiarism using any free online web tool such as Duplichecker (http://www.duplichecker.com/), etc. If the plagiarism level exceeds $10 \%$, the manuscript will be rejected without further processing for peer review. While submitting the manuscript check your submission for availability of following component:

1. Abstract (structured abstract for research paper and nonstructured abstract for clinical case reviews, clinical case reports and review articles)

2. Cover letter

3. Title page (email ID of all the authors and phone number should be provided) with contributes details

4. Conflict of interest

5. Title page of the manuscript

6. Manuscript page

7. Figures

8. Tables
9. Supplementary materials (ethical committee permission letter, additional publication materials, CONSORT statement, etc.)

Please note that, the manuscript which has improper/incomplete first page, poor language, improper reference style and citation will not be considered for review process/publication.

\section{From the Editor}

In past few months, due to transition from previous publisher, we have faced some difficulties in processing and handling of manuscript. From this point onwards, the preliminary report will be send to author in a week's time period (suitable for publication/not suitable for publication). The approx. review period will be around 30-45 days from the day of assigning the reviewers and final decision will be made based on subjects expects and reviewer's comments. The average manuscript processing time will be around 45-60 days [3]. Once final decision arrived, uncorrected author proof available in science direct in couple of week period from the date of acceptance. In future, we will highlight more about importance of peer review process.

We wish you happy reading of this issue.

"Having no data is risky, having wrong data/wrong interpretation is dangerous hence make value for your own true original data/interpretation by publishing your research work in leading scientific journals."

-Author

\section{References}

1. Author. Available form http://en.wikipedia.org/wiki/Author last Accessed on 22.05.13.

2. Parasuraman S. Journal of Young Pharmacists: now published with Elsevier. $J$ Young Pharm. 2013;5:1-2.

3. Parasuraman S. My vision: J Young Pharm. J Young Pharm. 2012;4:127-128.

Subramani Parasuraman* Editor-in-Chief, Journal of Young Pharmacist

K.K. Mueen Ahmed Former Editor-in-Chief, Journal of Young Pharmacist

* Corresponding author. Pharmacology Unit, Faculty of Pharmacy, Asian Institute of Medicine, Science and Technology (AIMST) University, Bedong-Semeling Road, Bedong 08100, Kedah, Malaysia. E-mail addresses: parasuraman@inpharm.org, jypeditor@gmail.com

Available online 10 July 2013 